\title{
Direct evidence for a role of working memory in the attentional blink
}

\author{
ELKAN G. AKYÜREK AND BERNHARD HOMMEL \\ Leiden University, Leiden, The Netherlands \\ AND \\ PiERre Jolicene \\ University of Montreal, Montreal, Quebec, Canada
}

\begin{abstract}
Theories of selective attention often have a central memory component, which is commonly thought to be limited in some way and is thereby a potential bottleneck in the attentional process. There have been only a few attempts to validate this assertion, and they have produced mixed results. This study presents a specific examination of the link between working memory and attention by engaging active rather than passive memory operations. Two experiments are reported that provide evidence for the involvement of working memory in the attentional blink (AB) phenomenon. Memory loads of increasing size had a detrimental effect on attentional performance within the blink-sensitive interval, but not beyond. Speeded response requirements proved to modulate the $\mathrm{AB}$, but were independent from the memory load effect. Theoretical implications for current models of selective attention are discussed.
\end{abstract}

When observers attempt to report two successive targets presented in a rapid serial visual stream of stimuli, the second target (T2) is often missed when it follows the first (T1) within an interval of $500 \mathrm{msec}$. The inability to report both targets under these circumstances is referred to as the attentional blink (AB; Raymond, Shapiro, \& Arnell, 1992). It is commonly assumed that the AB arises as a result of processing $\mathrm{T} 1$, which is thought to put $\mathrm{T} 2$ "on hold" and leave its representation vulnerable to decay. Chun and Potter (1995) proposed a two-stage model along these lines to account for the AB phenomenon (cf. Potter, Staub, \& O'Connor, 2002), which is also compatible with the somewhat broader central interference theory proposed by Jolicœur (1998).

The first stage of Chun and Potter's (1995) model consists of the (more or less rapid) detection of visual target stimuli on the basis of the defining sensory feature(s) or category membership. Targets (and distractors, depending on their similarity to targets) compete for detection to the degree that they match the detection criteria, especially when they appear in quick succession (i.e., at Lag 1, where T2 succeeds T1 directly). This competition may lead to confusion about the order of targets (Hommel \& Akyürek, 2005) or-given that representations at this stage are considered to be prone to mutual interference-the forgetting of one target (mostly T2; Potter et al., 2002). The early processes corresponding to this first stage of Chun and Potter's model are sensory and perceptual encoding in central interference theory.
The second stage is where further processing of selected stimulus representations takes place, as well as their transfer to short-term memory. This stage is thought to be capacity limited: When it is occupied by one target, the transfer of all subsequent stimuli from the first to the second stage is delayed, which makes their representations vulnerable to interference and decay - the more so the longer the second stage is occupied. This stage in the two-stage model is similar to the process of short-term consolidation (STC) in the central interference theory (Jolicœur, 1998; see also Jolicœur \& Dell' Acqua, 1998, 1999). In addition to STC, this theory also posits further cognitive processes, such as response selection, to be able to delay the processing of T2 and thus to contribute to the $\mathrm{AB}$.

There have been a number of studies investigating the specific effects of delays at various points in time over the course of attentional processing. For example, Chun and Potter (1995) demonstrated a positive correlation between the size of the $\mathrm{AB}$ and the discriminability of $\mathrm{T} 1$. When T1 was more similar to the distractors surrounding it (in particular, the distractor following it) in terms of visual features or categorical overlap, the AB was more pronounced. A similar result was obtained by Seiffert and Di Lollo (1997) for variations of the temporal and spatial relationship between the first target and its successor and by Crebolder, Jolicœur, and McIlwaine (2002) for signal probability. As outlined in the central interference theory, manipulations affecting processing of $\mathrm{T} 1$ before or in the $\mathrm{AB}$ bottleneck would be expected to carry forward and

E. G. Akyürek, akyurek@psy.Imu.de 
influence the accuracy of report of T2 (Jolicœur, 1998). Given that the above manipulations targeting the processing of $\mathrm{T} 1$ affected the $\mathrm{AB}$, these manipulations are likely associated with a locus situated at or before the attentional bottleneck. However, in another study focusing explicitly on the perceptual quality of the targets, McLaughlin, Shore, and Klein (2001) observed no relation between the difficulty of processing the first target and the AB. This result may serve as a reminder that some caution is warranted when trying to interpret carry-over effects that may bridge much of the attentional processes (i.e., those in both stages). According to the central interference theory, only manipulations affecting the duration of central or precentral processing of $\mathrm{T} 1$ will influence the accuracy of report of T2. A manipulation that affects accuracy of report of T1, but not the duration of processing of T1, such as a data-limiting effect of masking, should not carry forward onto processing of T2 and thus should have no effect on the AB. This may have occurred in the study of McLaughlin et al.

Other investigators have taken a slightly different approach, focusing more explicitly on "late" aspects of processing. Shapiro, Raymond, and Arnell (1994) manipulated identification difficulty by comparing performance across various set sizes of potential $\mathrm{T} 1$ identities (i.e., $\mathrm{T} 1$ set size). In one experiment, they compared a set size of 3 with one of 25 from an earlier experiment (Raymond et al., 1992). Somewhat disappointingly, strong evidence for a modulation of set size on the $\mathrm{AB}$ was not found. Shapiro et al. reported a nonsignificant main effect of set size and an interaction effect with intertarget lag, the latter of which was not supported by post hoc tests on the individual lag conditions. They concluded that target difficulty in this sense was not correlated with $\mathrm{AB}$ magnitude and hence not central to the origin of the $A B$, but also acknowledged that it might play a small role nonetheless.

Focusing more on short-term memory consolidation than on identification difficulty, Akyürek and Hommel (2005, 2006) found that (a lack of) short-term memory storage capacity has only a limited effect on the AB. This was a surprising finding, because the two-stage model positions memory consolidation at the supposedly critical capacitylimited second stage responsible for the $\mathrm{AB}$. In the experiments of Akyürek and Hommel (2005, 2006), participants were presented with a dual-task paradigm in which a set of items had to be held in memory (on a per-trial basis) for the duration of an attentional task designed to elicit an AB. When the memory set increased in size or when the items in the memory set were related to those in the attentional task, task performance decreased. But since this drop was essentially constant across all T1-T2 lags, it was unlikely to relate to the bottleneck underlying the $\mathrm{AB}$.

Summarizing, the research to date has shown some clear modulations of the $\mathrm{AB}$, in particular those studies involving the discriminability and predictability of stimuli. Some mixed results have also been found, especially from the studies involving perceptual quality (masking) or short-term memory. A caveat is important at this point. The studies of Shapiro et al. (1994) and Akyürek and Hommel $(2005,2006)$ were aimed at memory-related fac- tors, but may have posed a rather conservative test. First, the set size condition employed by Shapiro et al. was realized between groups and done in an all-or-none fashion. That is, one group of participants knew in advance that the target was one out of three possible letters for the duration of the entire experimental session, whereas the other group was instructed with the larger set of 25 letters. This method of presenting a fixed set of alternatives may lead to a form of task adaptation or microlearning, especially if the set consists of very familiar stimuli (i.e., the alphabet) that allow relatively effortless matching with the task set via chunking, familiarity, and repetition effects. In other words, once participants have adapted to the experimental conditions, it is not clear whether the effort of matching the identity of a stimulus against the range of items in the set should be strongly correlated with the size of that set. One possible avenue for future research is to test this more directly by using reaction time (RT) measures or eventrelated potentials. If this correlation was indeed lacking under these rather static task circumstances, then there may have been little reason to expect differences between the two set sizes that Shapiro et al. employed.

Second, the experiments of Akyürek and Hommel $(2005,2006)$ were primarily focused on memory capacity in terms of storage capacity. It is possible that although (consolidation of representations in) working memory is crucial to attention, the bottleneck revealed by the $\mathrm{AB}$ is not due to a lack of storage capacity even when items are closely related. In terms of the two-stage model, this holds that processing in the second stage may not be limited by a shortage of memory capacity. Instead, the number of active memory processes - such as scanning and updating of items in memory - that have to be executed during the task at hand may form the bottleneck. In effect, then, the bottleneck of consolidation in memory may be processing, rather than storage, capacity. Recent research has demonstrated that a relatively passive memory process, such as maintenance of items, may be fundamentally different from active consolidation (Jolicœur \& Dell' Acqua, 1998; Woodman \& Vogel, 2005) or manipulation (Han \& Kim, 2004). This supports the notion that attention may in turn be affected differently by consolidation as opposed to maintenance processes.

At present, it is still unclear what the impact of memory processing is on the extent of the $\mathrm{AB}$, since the previous studies did not engage these processes. The present study was designed to address the concerns raised above and investigate the importance of memory processes (in the sense of STC; Jolicœur, 1998) in the emergence of the AB. To this end, a Sternberg task (Sternberg, 1966) with a variable memory set size was implemented on a per-trial basis. Participants were asked to encode a memory set on each trial and use it to match T1 from an otherwise typical rapid serial visual presentation (RSVP) to the item(s) in the memory set, which varied in size from 1-4 items. That is, the memory set was not only to be maintained for later report, as was the case in the experiments of Akyürek and Hommel (2005, 2006), but actively accessed during the RSVP task. We predicted that the resulting scanning process would affect attentional performance, in contrast 
to what was observed previously for merely maintaining items in memory.

\section{EXPERIMENT 1}

To examine the role of active working-memory processes in the $\mathrm{AB}$, a different random set of one to four letters was presented at the start of each trial. Participants were then asked to successively identify a highlighted letter (T1) and a single digit (T2) in an RSVP stream of letters. T2 could appear either quickly after T1 (i.e., at lag 3 , with two distractors in between $\mathrm{T} 1$ and T2) or much later (at lag 8). The first lag can be expected to fall right into the $\mathrm{AB}$, whereas the second one should be well outside the AB (see, e.g., Raymond et al., 1992). The report of T1 was tied to the memorized set; participants had to determine whether the highlighted letter was or was not a member of the memorized set. Along the lines of Jolicœur's (1998) central interference theory, a larger set should lengthen the time spent scanning the memory set, which would delay the consolidation of the outcome of the central processing required to complete Task 1 . This should delay the transfer of $\mathrm{T} 2$ to the second stage and thus expose $\mathrm{T} 2$ to longer-lasting interference if T2 appears at a shorter lag than $\mathrm{T} 1$. If so, the $\mathrm{AB}$ - expressed as the impairment of performance on $\mathrm{T} 2$ at the short lag in comparison with the long lag-should increase with set size. To provide a direct estimate of the impact of set size on the time to process $\mathrm{T} 1$, we asked participants to carry out speeded responses to T1. Doing so also effectively ensured that the matching operations between $\mathrm{T} 1$ and the memory set were carried out immediately - as the RSVP stream unfolded and as T2 processing required access to capacitylimited mechanisms - rather than after the termination of the RSVP stream. In the latter case, participants would merely encode $\mathrm{T} 1$ and perform the match to the memory set after T2 had already been encoded, which would defeat the logic of the experiment (see Jolicœur, 1999). Experiment 2 tested whether and how the speeded response in Task 1 might have affected the outcome.

\section{Method}

Design. The experiment used a factorial design that was created by crossing two variables: memory set size for the T1 and T1-T2 lag. Memory set size had four levels: one, two, three, or four letters. Lag - that is, the temporal distance between T1 and T2 in the RSVP stream-was either three or eight. Dependent measures in the repeated measures within-subjects ANOVA were accuracy for T1, reaction time to $\mathrm{T} 1$, and accuracy for $\mathrm{T} 2$, given that $\mathrm{T} 1$ was correctly identified (T2|T1).

Participants. Twenty Leiden University students (15 female, 5 male) participated for pay or course credit. The mean age was 20.7 years. All participants reported normal or corrected-to-normal vision. The data from one female participant were excluded from analysis because no valid response to T1 was logged.

Apparatus and Procedure. Participants were seated individually in a small, dimly lit room. Stimulus presentation was controlled by the PST E-Prime runtime component using the Intel i815 chipset onboard graphics system of an Intel Pentium III computer. Stimuli were displayed on a 17-in. cathode-ray tube monitor driven at a resolution of $800 \times 600$ pixels at $75 \%$ contrast and brightness settings, a 16 bits color depth, and a refresh rate of $100 \mathrm{~Hz}$. Average viewing distance was approximately $50 \mathrm{~cm}$, but it was not fixed. A single session consisted of 512 experimental and 16 practice trials, taking about one hour to complete on average.

Trials were self-paced and initiated by pressing the "Enter" key on the keyboard. After a blank interval of $200 \mathrm{msec}$, the memory set was presented centrally in blue for $2,000 \mathrm{msec}$ on a light gray background that remained constant throughout the trial. After another 200-msec delay, a red fixation mark ("+") appeared in the center of the screen for $150 \mathrm{msec}$, which was in turn followed by a 50 -msec blank. An RSVP of 20 stimuli ensued. Eighteen of these were black capital distractor letters in 18-pt. bold Courier New font. T1 was a letter similar to the distractors, but was colored lime green. T2 was a digit in the same color and font as the distractors. Each stimulus remained on screen for $50 \mathrm{msec}$ and was followed by a $50-\mathrm{msec}$ blank. Finally, after a $200-\mathrm{msec}$ pause, the T2 response screen was presented. While the identity of T2 was queried and its response given at leisure, the response to T1 was to be given as quickly as possible upon perception of the target. Identification of T2 was accomplished by pressing the corresponding digit on the keyboard. T1 required a judgment of a match with the items in the memorized set. A key labeled with a black sticker represented a no-match response (T1 not in the memory set), and a white-labeled key represented the opposite (T1 in the memory set). Participants were required to keep their index fingers on these keys to enable a quick reaction. The underlying keys were Q (white) and P (black). All responses were logged on a standard USB keyboard $(125 \mathrm{~Hz})$

Letter stimuli were chosen randomly from the full alphabet with the constraint that no letter was repeated within a trial with the exception of the identity of $\mathrm{T} 1$ if it matched a letter from the memory set, which was the case on $50 \%$ of the trials. The digit stimulus was chosen randomly from the digits $2-9$. T1 onset varied randomly between the fifth and seventh temporal position in the stream, with positions equally distributed across trials. $\mathrm{T} 2$ onset followed $\mathrm{T} 1$ at a lag of three (two intervening items, T1-T2 SOA of $300 \mathrm{msec}$ ) or eight (seven intervening items, T1-T2 SOA of $800 \mathrm{msec}$ ).

\section{Results and Discussion}

The mean accuracy in Task 1 (memory-scanning task) for each memory set size and each T1-T2 lag is shown in Figure 1 (left panel). The ANOVA showed a significant main effect of memory set size $\left[F(3,54)=30.76, M S_{\mathrm{e}}=\right.$ $.004, p<.001]$. The increase of set size resulted in a decrease in T1 accuracy from $85 \%$ correct to $72.6 \%$, in a fairly linear pattern. There was no effect of T2 $\operatorname{lag}(F<1)$, or of the interaction of both variables $[F(3,54)=1.06$, $\left.M S_{\mathrm{e}}=.002, p<.38\right]$.

Also shown in Figure 1 (right panel) are the mean RTs for the various combinations of memory set size and lag. The ANOVA of T1 RT showed only a main effect of set size $\left[F(3,54)=100.76, M S_{\mathrm{e}}=836.4, p<.001\right]$. Consistent with previous observations (see, e.g., Sternberg, 1966), an increase in set size led to longer RTs, suggesting that memory search varies as a function of the number of comparisons to be made. Although there was a steady increase in RTs from one $(614 \mathrm{msec})$ to four $(722 \mathrm{msec})$ items, the differences were most pronounced between the smallest memory sets. The T2 lag variable and the interaction term did not reach significance $\left[F(1,18)=2.28, M S_{\mathrm{e}}=356.3\right.$, $p<.15$, and $F(3,54)=2.19, M S_{\mathrm{e}}=770.1, p<.10$, respectively]. Although these $p$ values might be taken to represent a (weak) trend, their relevance was limited to an effect size of less than $5 \mathrm{msec}$ for T2 lag and $13 \mathrm{msec}$ for the interaction, both of which paled in comparison with the magnitude of the effect of memory set size. In any case, the 


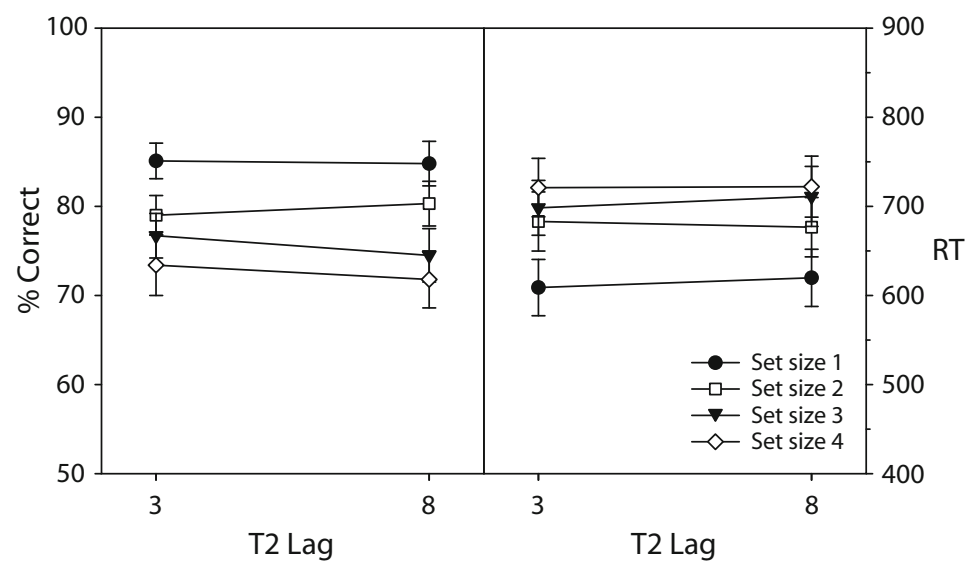

Figure 1. Experiment 1. Task 1 accuracy (percent correct, left panel) and reaction time (in milliseconds, right panel), plotted as a function of $T 2$ lag and memory set size. Error bars indicate $\pm 1 S E$.

strong memory set size effect on $\mathrm{T} 1$ performance confirms that our major manipulation worked: T1 processing time increased with set size, suggesting that memory operations took longer the larger the set. The question remains as to whether this affected the size of the AB.

Figure 2 shows the means of T2 identification accuracy (given that a correct response was made in Task 1). The ANOVA showed effects of both variables of memory set size and lag, as well as a significant interaction between them. An increase in memory set size diminished T2 accuracy $\left[F(3,54)=14.61, M S_{\mathrm{e}}=.005, p<.001\right]$, which was similar to the pattern found in the analysis of T1 accuracy. The short $\mathrm{T} 2 \mathrm{lag}$, in which $\mathrm{T} 2$ was the third item after $\mathrm{T} 1$, resulted in particularly poor performance $[F(1,18)=$ $\left.101.36, M S_{\mathrm{e}}=.049, p<.001\right]$. On average, performance dropped from $95 \%$ at the long lag to $59 \%$ at the short lag, which indicates a sizeable AB. Most importantly, for our purposes, the lag effect was modulated by memory set size $\left[F(3,54)=17.39, M S_{\mathrm{e}}=.004, p<.001\right]$. Memory set size had a strong impact on performance at the short but not at the long lag. In other words, the $A B$ increased with set size, ranging from a rather mild drop of a little more than $20 \%$ with a load of a single memorized stimulus to one of double the size for the four-item load. Although performance for two- and three-item sets was between that for the other conditions at $57.4 \%$ and $58.3 \%$, respectively, no difference between them was apparent. That might have occurred for two reasons: (1) some structural cognitive restraint that takes effect only after more than three items are presented, or (2) the fact that chunking twoand three-letter combinations is approximately equally difficult. The latter might originate in the prevalence of two- and three-letter acronyms in common (Dutch) language. In any case, the principal outcome was the modulation of the $\mathrm{AB}$ by the size of the memorized set.

\section{EXPERIMENT 2}

Experiment 1 provided much clearer evidence for an interaction between working memory processes and the
AB than the previous studies of Shapiro et al. (1994) and Akyürek and Hommel $(2005,2006)$. We attribute this discrepancy to the fact that our present task design required active scanning of the contents of working memory at the time that T2 was presented. However, we also introduced another design element that might have been responsible for the more pronounced interaction between working memory and AB. To test whether our main manipulation (of working memory set size) worked as expected, we required participants to perform speeded responses to T1, whereas the responses to both targets were unspeeded in the studies of Shapiro et al. and Akyürek and Hommel (2005, 2006). On the one hand, there is ample evidence that the bottleneck that underlies the $\mathrm{AB}$ and response selection have a lot in common (Jolicœur \& Dell' Acqua, 2000), but, on the other hand, there is also evidence that speeding or not speeding responses in an AB context makes a difference (see, e.g., Jolicœur, 1998). In particu-

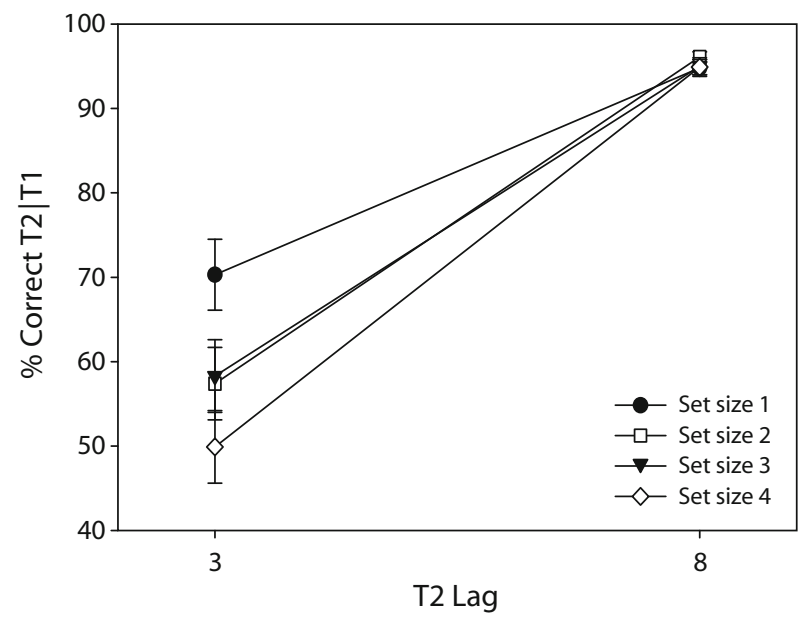

Figure 2. Experiment 1. Identification accuracy (percent correct) for $T 2$, given that the response to $T 1$ was correct, for each T1-T2 lag and memory set size. Error bars indicate $\pm 1 S E$. 


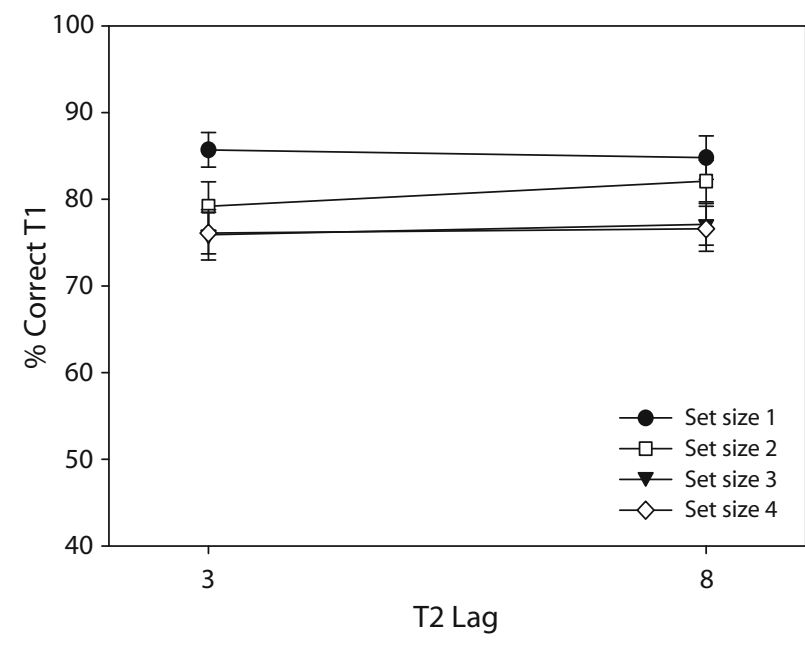

Figure 3. Experiment 2. Task 1 accuracy (percent correct) as a function of T1-T2 lag and memory set size. Error bars indicate $\pm 1 S E$.

lar, Jolicœur (1999) found that T2 performance is affected by the number of $\mathrm{T} 1$ response alternatives if $\mathrm{T} 1$ responses are speeded, but not if they are unspeeded. At the same time, it is unlikely that response selection difficulty was affected by memory set size (Sternberg, 1966), which means that memory scanning should have been responsible for the interference with the consolidation of $\mathrm{T} 2$. Still, we wanted to be sure that the findings of our present Experiment 1 generalize to the more common RSVP task with unspeeded responses. To make sure that they do, we repeated Experiment 1, with the only difference being that the response to $\mathrm{T} 1$ was no longer speeded.

\section{Method}

Another twenty Leiden University students (18 female, 2 male) with normal or corrected-to-normal vision participated for pay or course credit. None of them had participated in Experiment 1. The mean age was 19.7 years. The design was identical to that of Experiment 1, except that Task 1 (i.e., the response to T1) was no longer speeded and no RT was measured. Participants were instructed to respond at leisure to T1 after the RSVP offset. A response screen was presented at that moment for this purpose. After responding to this first query, the experiment continued as before with the T2 response screen.

\section{Results and Discussion}

As shown in Figure 3, accuracy in Task 1 was significantly affected by memory set size $[F(3,57)=33.18$, $\left.M S_{\mathrm{e}}=.002, p<.001\right]$. The effect represented a uniform (but not quite linear) decrease of T1 performance for each increase in memory set size. The effect of T2 lag did not reach significance $\left[F(1,19)=1.02, M S_{\mathrm{e}}=.003, p<.33\right]$, and neither did the interaction with set size $[F(3,57)=$ $\left.1.26, M S_{\mathrm{e}}=.002, p<.30\right]$. These results were virtually identical to those of Experiment 1.

The means for T2 identification accuracy are plotted in Figure 4. Report accuracy for T2 showed significant main effects of memory set size $[F(3,57)=22.39$, $\left.M S_{\mathrm{e}}=.003, p<.001\right]$ and $\mathrm{T} 1-\mathrm{T} 2 \operatorname{lag}[F(1,19)=65.71$,
$\left.M S_{\mathrm{e}}=.035, p<.001\right]$. Increasing memory set size decreased T2 performance in a fashion similar to that in Experiment 1, with the steepest drops occurring between loads 1 versus 2, and 3 versus 4 . Lag had the predictable effect of causing low T2 performance at lag $3(69.1 \%)$ compared with lag 8 (92.9\%). Again, there was a sizable AB. Most importantly, this lag effect was modified by set size $\left[F(2,38)=6.45, M S_{\mathrm{e}}=.048, p<.005\right]$. Because of a significant test of sphericity, the degrees of freedom for this test were Greenhouse-Geisser corrected. A Tukey's pairwise test was done on the means at lag 8 to confirm the absence of an effect of set size there; no pair was significant $[q(4,19)=3.98, t<2.81]$.

This pattern nicely replicates our findings from Experiment 1: Memory set size strongly affected performance at lag 3 but not at lag 8. An ANOVA on the combined results of both experiments (included as a between-subjects variable) revealed only a single interaction involving experiment: The two-way interaction of experiment and lag $\left[F(1,37)=7.17, M S_{\mathrm{e}}=.042, p<.05\right]$ indicated that the $\mathrm{AB}$ was more pronounced in Experiment 1 than in Experiment 2 . That is, the $\mathrm{AB}$ was indeed modulated by the response requirements of $\mathrm{T} 1$, which confirms that response selection has a part in the bottleneck giving rise to the $\mathrm{AB}$ (Jolicœur, 1998). Most important for present purposes, however, was that the response requirement (i.e., the experiment) did not modify the interaction of lag and memory load $\left[F(3,111)=1.16, M S_{\mathrm{e}}=.005, p<.33\right]$. That is, the $\mathrm{AB}$ is modulated by set size to the same extent whether the response to $\mathrm{T} 1$ was speeded or unspeeded.

\section{GENERAL DISCUSSION}

The present experiments have demonstrated a clear modulation of the $\mathrm{AB}$ phenomenon by concurrent scanning (Sternberg, 1966) of a small set of letters held in shortterm memory. Scanning a larger memory set (e.g., four

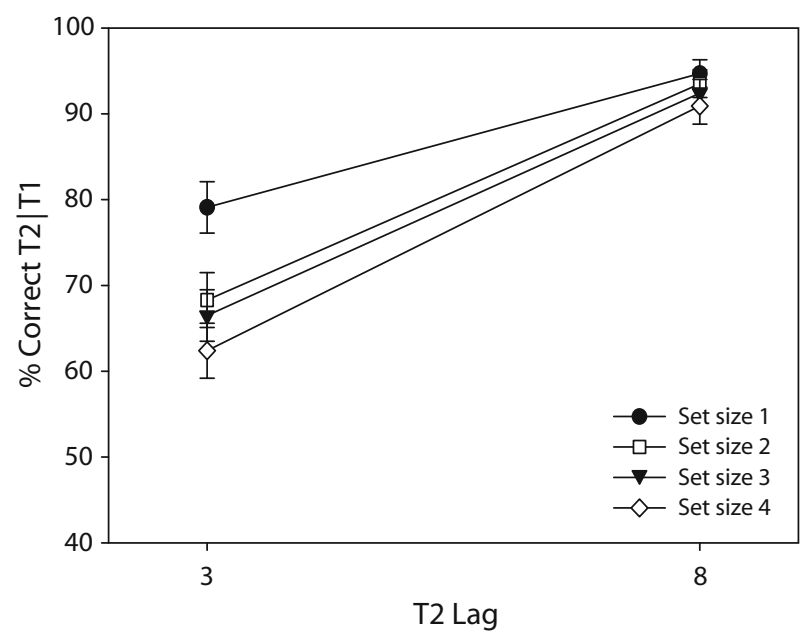

Figure 4. Experiment 2. Identification accuracy (percent correct) for $\mathrm{T} 2$, given a correct response in Task 1, for each T1-T2 lag and memory set size. Error bars indicate $\pm 1 S E$. 
items) took longer and produced a larger $\mathrm{AB}$ than scanning a smaller memory set (e.g., one item). Our experiments are thus the first to demonstrate a direct link between the mechanisms that mediate dynamic processing in short-term memory and those involved in the AB phenomenon.

As was demonstrated by Shapiro et al. (1994), simply varying the size of the selection sets for T1 is not sufficient to have much of an effect on Task 2 success rates. The set size manipulation of Shapiro et al. was much larger than the present one ( 3 vs. 25 as compared with 1-4), which rules out the possibility that their manipulation was less demanding in terms of absolute memory load.

Experiment 2 addressed another potentially relevant difference between the experiments of Shapiro et al. and the present Experiment 1 by showing that whetherT1 responses are speeded or unspeeded is of little consequence for the impact of working memory on the AB. It therefore seems more likely that the crucial ingredient is the requirement to actively scan varying set sizes in the present paradigm. When T1 was selected, the memory set was scanned, even when an immediate response was not needed. It is this scanning process that takes its toll on the efficiency of attention, reducing the likelihood of accurate $\mathrm{T} 2$ report. Note that the need to perform a quick response did affect the magnitude of the blink (Jolicœur, 1998), but it was also clearly dissociable from the modulation caused by the memory task, consistent with Sternberg's evidence suggesting separate memory scanning and response selection stages.

It is not clear why participants in Experiment 2 (delayed response in Task 1) did not only encode T1 during the RSVP stream and perform the scan of the memory set only afterwards, when time-critical operations required to process $\mathrm{T} 2 \mathrm{had}$ been competed. In previous work that investigated manipulations of the difficulty of response selection, participants were able to postpone response selection when it was advantageous to do so. In this case, response-selection difficulty had an effect on the $A B$ when Task 1 was speeded, but had no effect when Task 1 was not speeded and response selection was postponed (Jolicœur, 1999). Either participants in Experiment 2 were not aware of the increased cost of performing the memory scan immediately or they were not able (or found it difficult) to postpone the memory scan.

Akyürek and Hommel $(2005,2006)$ showed that the storage capacity of short-term memory does not impose an attentional problem in the $\mathrm{AB}$ paradigm. In some of their experiments, the load on memory was considerably higher than that in the present study (e.g., up to six symbol characters), and yet, virtually no impact on the AB was observed. However, even though memory performance attested that holding these items was very difficult, there was no need to process them actively during the RSVP stream. Together with the present observation of pronounced memory set size effects, these findings support the idea that it is not so much the number of slots in working memory that is the limiting factor in multitasking, but the number of processes that can access the contents of working memory simultaneously. Further support for this idea comes from studies showing that in comparison with the rather mild impact of memory-maintenance op- erations on task performance (Hommel \& Eglau, 2002; Logan, 1978, 1979, 1980), working memory updating operations can exert strong and damaging side effects, such as those revealed by the $n$-back task (Cohen et al., 1994). This again fits well with the observation that updating and maintenance functions have different neuroanatomical bases (Smith \& Jonides, 1998) and that the dorsolateral prefrontal cortex - assumed to play a key role in the more active working memory functions-is much more activated in updating tasks than in maintenance tasks (Cohen et al., 1994; Smith, Jonides, \& Koeppe, 1996).

Demonstrations of the effect of T1 difficulty on T2 accuracy that are focused on the (local) discriminability or encoding difficulty of $\mathrm{T} 1$ have proven that processes in the first stage of attention can cause the $A B$, since their effects carry over to "late" processes (cf. Jolicœur, 1998; Seiffert \& Di Lollo, 1997). By increasing the amount of time the first target spends in the first stage and, hence, by delaying its entrance to the second stage, the second target suffers. The present results complement this picture by demonstrating that memory scanning - a process that affects the second stage, or STC of T1-also delays the processing of T2. The limits on the second stage, as observed by the modulation of attentional performance by memory set size, show that at least part of the capacity limitations of this stage are found in processing, rather than in storage capacity.

The present results also support Olson, Chun, and Anderson's (2001) proposed explanation for the modulation of the $\mathrm{AB}$ by phonological word length. In their experiments, a more severe $\mathrm{AB}$ was found for phonologically longer T1 words, even when they were controlled for typographic length. They suggested that the phonological word length effect was due to a direct effect of this length on the memory encoding process. Olson et al. conceded that alternative explanations for their data, such as an independent subvocalization routine drawing resources from $\mathrm{T} 1$ encoding, could not be fully discarded. Although interactions such as this may still exist, the presently observed modulation of the AB by the difficulty (or length) of the encoding process fits nicely with the account that Olson et al. proposed.

In summary, the present work highlights the importance of dynamic memory operations in the $\mathrm{AB}$ paradigm and of the distinction between two notions of "capacity": processing capacity versus storage capacity. With regard to working memory, storage capacity refers to the amount of information that can be held in the store. Processing capacity refers to the amount of information that can be dealt with in a certain amount of time and to the consequences of engaging a mechanism or process on other ongoing cognitive activities. This latter notion of processing capacity is often assessed in dual-task paradigms by measuring the impact of processing in one task on performance in another task. Filling up working memory to different degrees addresses one of the limitations of working memory-namely, the limited amount of storage space in the memory store. Holding the information in the store once it is there, however, appears to require relatively little processing capacity (Jolicœur \& Dell'Acqua, 1998; 
Logan, 1978) and is broadly consistent with a recent finding using the $\mathrm{AB}$ paradigm (Akyürek \& Hommel, 2005, 2006). Interestingly, a similar pattern of results have been shown recently in the domain of visual search by Woodman, Vogel, and Luck (2001) and Han and Kim (2004). Woodman et al. employed a dual task design in which participants had to perform a visual search task while either holding items in visual working memory or not. Although they found an overall effect of task difficulty, search efficiency over different set sizes remained virtually identical for each memory condition. Han and Kim used a slightly different dual task paradigm in which participants had to either hold digits or letters in memory or manipulate them (counting backward, reordering alphabetically) while performing a visual search task. They observed that the maintenance of items in memory did not affect search efficiency, but that the manipulation of these same items had a large effect, progressively deteriorating search performance for larger sets.

Accessing working memory, or scanning a memory set, is perhaps the paradigmatic capacity-limited operation involving interactions with the contents of working memory, because this operation appears to impose a seriality of processing that is difficult to overcome (Shiffrin \& Schneider, 1984; Sternberg, 1966). Results from work using the psychological refractory period (PRP) paradigm (see, e.g., Heil, Wahl, \& Herbst, 1999) are consistent with the hypothesis that memory scanning can postpone or slow down other capacity-demanding operations. The present work, which demonstrates that scanning the contents of working memory strongly modulates the $\mathrm{AB}$ effect, extends this and earlier work (see, e.g., Jolicœur, 1998, 1999; Jolicœur, Sessa, Dell' Acqua, \& Robitaille, 2006) linking the $A B$ phenomenon to general central capacity limitations in the human cognitive architecture.

\section{AUTHOR NOTE}

Address correspondence to E. G. Akyürek, Ludwig-MaximiliansUniversität Munich, Experimental Psychology, Leopoldstraße 13, 80802 Munich, Germany (e-mail: akyurek@psy.lmu.de).

\section{REFERENCES}

AKyÜrek, E. G., \& Hommel, B. (2005). Short-term memory and the attentional blink: Capacity versus content. Memory \& Cognition, 33, 654-663.

AKyÜReK, E. G., \& Hommel, B. (2006). Memory operations in rapid serial visual presentation. European Journal of Cognitive Psychology, 18, 520-536.

Chun, M. M., \& Potter, M. C. (1995). A two-stage model for multiple target detection in rapid serial visual presentation. Journal of Experimental Psychology: Human Perception \& Performance, 21, 109-127.

Cohen, J. D., Forman, S. D., Braver, T. S., Casey, B. J., ServanSchreiber, D., \& Noll, D. C. (1994). Activation of prefrontal cortex in a nonspatial working memory task with functional MRI. Human Brain Mapping, 1, 293-304.

Crebolder, J. M., Jolicceur, P., \& Mcilwaine, J. D. (2002). Loci of signal probability effects and of the attentional blink bottleneck. Journal of Experimental Psychology: Human Perception \& Performance, 28, 695-716.

Han, S.-H., \& Kim, M.-S. (2004). Visual search does not remain efficient when executive working memory is working. Psychological Science, 15, 623-628.

Heil, M., Wahl, K., \& Herbst, M. (1999). Mental rotation, memory scanning, and the central bottleneck. Psychological Research, 62, 48-61.

Hommel, B., \& AkyÜreK, E. G. (2005). Lag-1 sparing in the attentional blink: Benefits and costs of integrating two events into a single episode. Quarterly Journal of Experimental Psychology, 58A, 1415-1433.

Hommel, B., \& EgLaU, B. (2002). Control of stimulus-response translation in dual-task performance. Psychological Research, 66, 260-273.

JolicceUR, P. (1998). Modulation of the attentional blink by on-line response selection: Evidence from speeded and unspeeded Task decisions. Memory \& Cognition, 26, 1014-1032.

JoLICEUR, P. (1999). Concurrent response-selection demands modulate the attentional blink. Journal of Experimental Psychology: Human Perception \& Performance, 25, 1097-1113.

Jolicceur, P., \& Dell'AcQua, R. (1998). The demonstration of shortterm consolidation. Cognitive Psychology, 36, 138-202.

Joliceur, P., \& Dell'ACQUA, R. (1999). Attentional and structural constraints on visual encoding. Psychological Research, 62, 154-164.

Jolicceur, P., \& Dell'Acqua, R. (2000). Selective influence of second target exposure duration and Task ${ }_{1}$ load effects in the attentional blink phenomenon. Psychonomic Bulletin \& Review, 7, 472-479.

Joliceur, P., Sessa, P., Dell'Acqua, R., \& Robitaille, N. (2006). On the control of visual spatial attention: Evidence from human electrophysiology. Psychological Research, 70, 414-424.

LoGAN, G. D. (1978). Attention in character classification: Evidence for the automaticity of component stages. Journal of Experimental Psychology: General, 107, 32-63.

LOGAN, G. D. (1979). On the use of a concurrent memory load to measure attention and automaticity. Journal of Experimental Psychology: Human Perception \& Performance, 5, 189-207.

LoGan, G. D. (1980). Short-term memory demands of reaction time tasks that differ in complexity. Journal of Experimental Psychology: Human Perception \& Performance, 6, 375-389.

McLaughlin, E. N., Shore, D. I., \& Klein, R. M. (2001). The attentional blink is immune to masking-induced data limits. Quarterly Journal of Experimental Psychology, 54A, 169-196.

Olson, I. R., Chun, M. M., \& Anderson, A. K. (2001). Effects of phonological length on the attentional blink for words. Journal of Experimental Psychology: Human Perception \& Performance, 27, 1116-1123.

Potter, M. C., Staub, A., \& O'Connor, D. H. (2002). The time course of competition for attention: Attention is initially labile. Journal of Experimental Psychology: Human Perception \& Performance, 28, $1149-1162$

Raymond, J. E., Shapiro, K. L., \& Arnell, K. M. (1992). Temporary suppression of visual processing in an RSVP task: An attentional blink? Journal of Experimental Psychology: Human Perception \& Performance, 18, 849-860.

Seiffert, A. E., \& Di Lollo, V. (1997). Low-level masking in the attentional blink. Journal of Experimental Psychology: Human Perception \& Performance, 23, 1061-1073.

Shapiro, K. L., Raymond, J. E., \& Arnell, K. M. (1994). Attention to visual pattern information produces the attentional blink in rapid serial visual presentation. Journal of Experimental Psychology: Human Perception \& Performance, 20, 357-371.

ShifFrin, R. M., \& SchNeIder, W. (1984). Automatic and controlled processing revisited. Psychological Review, 91, 269-276.

SMith, E. E., \& Jonides, J. (1998). Neuroimaging analyses of human working memory. Proceedings of the National Academy of Science, 95, 12061-12068.

Smith, E. E., Jonides, J., \& Koeppe, R. A. (1996). Dissociating verbal and spatial working memory using PET. Cerebral Cortex, 6, 11-20.

Sternberg, S. (1966). High-speed scanning in human memory. Science, 153, 652-654.

WoOdMan, G. F., \& Vogel, E. K. (2005). Fractionating working memory: Consolidation and maintenance are independent processes. Psychological Science, 16, 106-113.

Woodman, G. F., Vogel, E. K., \& LucK, S. J. (2001). Visual search remains efficient when visual working memory is full. Psychological Science, 12, 219-224.

(Manuscript received November 7, 2005; revision accepted for publication February 14, 2006.) 